\title{
The Transmission of Mycobacterium tuberculosis in High Burden Settings
}

Tom A. Yates (MSc) 1,2§*, Palwasha Y. Khan (MBBCh) 3,4,5\$, Gwenan M. Knight (PhD) 5, 6,7, Jonathon G. Taylor (PhD) 8, Prof Timothy D. McHugh (PhD) 9, Marc Lipman (MD) 10, Richard G. White (PhD) 5,6, Ted Cohen (MD) 11, Prof Frank G. Cobelens (PhD) 12, 13, Prof Robin Wood (D.Sc(Med)) 3, 5, 14, Prof David A. J. Moore (MD) 5, 15, Prof Ibrahim Abubakar (FRCP) 1,16

1. Centre for Infectious Disease Epidemiology, Research Department of Infection and Population Health, University College London, London, UK.

2. Wellcome Trust Africa Centre for Population Health, Mtubatuba, South Africa.

3. Department of Infectious Disease Epidemiology, Faculty of Epidemiology and Population Health, London School of Hygiene and Tropical Medicine, London, UK.

4. Karonga Prevention Study, Chilumba, Malawi.

5. TB Centre, London School of Hygiene and Tropical Medicine, London, UK.

6. TB Modelling Group, London School of Hygiene and Tropical Medicine, London, UK.

7. National Institute for Health Research Health Protection Research Unit in Healthcare Associated Infection and Antimicrobial Resistance, Imperial College London, London, UK

8. UCL Institute for Environmental Design and Engineering, Bartlett School of Environment, Energy and Resources, University College London, London, UK.

9. Centre for Clinical Microbiology, University College London, London, UK.

10. Division of Medicine, University College London, London, UK.

11. Department of Epidemiology of Microbial Diseases, Yale School of Public Health, New Haven, CT, USA.

12. Department of Global Health, Academic Medical Center, Amsterdam, the Netherlands.

13. KNCV Tuberculosis Foundation, The Hague, the Netherlands.

14. The Desmond Tutu HIV Centre, Institute of Infectious Disease and Molecular Medicine, University of Cape Town, Cape Town, South Africa

15. Department of Clinical Research, London School of Hygiene and Tropical Medicine, London, UK.

16. MRC Clinical Trials Unit at UCL, University College London, London, UK

$\S$ These authors contributed equally to this work.

*Dr Tom Yates, t.yates@ucl.ac.uk.

Unacceptable levels of Mycobacterium tuberculosis transmission are observed in high burden settings and a renewed focus on cutting person-to-person transmission in these communities is needed. Here, we review recent developments in our understanding of airborne transmission. We outline approaches to measuring transmission in populations and trials and we describe the Wells-Riley Equation, which is used to estimate transmission risk in indoor spaces. Current research priorities include identifying effective tuberculosis infection control strategies; better understanding where transmission occurs and the transmissibility of drug resistant strains; and estimating the impact of HIV and antiretroviral therapy on transmission dynamics. When planning research and designing interventions to interrupt transmission, resource constraints common in high burden settings, including shortages of healthcare workers, must be considered. 


\section{Introduction}

Sustained declines in disease incidence of up to $20 \%$ per year are required to meet the targets set out in the World Health Organization (WHO) End-TB Strategy. ${ }^{1,2}$ However, incidence is only estimated to be declining at $1.5 \%$ per annum. ${ }^{3}$ This trend is consistent with model predictions regarding the likely impact of current control strategies, ${ }^{4}$ which focus on case detection and treatment completion. ${ }^{5}$ Even in areas with good rates of case finding and treatment completion, evidence suggests that transmission remains a problem. Although quality data on active tuberculosis (TB) in children under the age of 5 years are limited, paediatric cases indicate continuing high levels of transmission.3, 6, 7 Recent tuberculin surveys in highprevalence countries estimate annual risks of Mycobacterium tuberculosis (MTB) infection of 0.3-2.2\%, ${ }^{8-12}$ but exceeding five per cent in some parts of southern Africa. ${ }^{13,14}$ Test reversions (negative tests in people who previously had a positive test) mean such cross sectional surveys may underestimate transmission. ${ }^{15}$ Data on MTB transmission derived using molecular typing methods from high burden areas are limited to a small number of research active settings. Nevertheless, these data suggest more disease results from recent transmission than from reactivation of latent $\mathrm{TB},{ }^{16}, 17$ particularly in HIV positive people. ${ }^{18}$ The rapid rebound in TB incidence following the discontinuation of isoniazid preventive treatment (IPT) in recent Southern African studies suggest on-going transmission remains important in high burden settings, ${ }^{19}, 20$ although models predict a contribution from reactivation disease implying IPT may not sterilise. ${ }^{21,22}$

To achieve the goals of the End-TB strategy, ${ }^{2}$ an increased emphasis on reducing person-to-person MTB transmission in high burden settings is needed. This review summarises recent research on MTB transmission in these settings. We focus on the biology of airborne MTB transmission, measuring transmission in populations, and modelling transmission using the Wells-Riley approach. We conclude by identifying research priorities. We do not discuss transmission-blocking vaccines nor mixed infections, each the subject of a recent review article. ${ }^{23,}{ }^{24} \mathrm{It}$ is important to note that there is no international consensus on TB incidence or prevalence thresholds that constitute 'high burden', although a TB incidence of 100 per 100,000 per year has recently been used by the WHO. ${ }^{25}$ Most of the studies we review were conducted in communities with a TB incidence of 100 per 100,000 per year or more.

\section{Airborne MTB transmission}

Whilst MTB complex organisms can be spread via unpasteurised milk, direct inoculation and other means, we focus on the predominant route, airborne transmission. The fundamentals of airborne MTB transmission were described by William Frith Wells, Richard Riley, Robert Loudon, Rena Roberts, and others, more than sixty years ago. ${ }^{26}$ Although much remains unknown, progress in basic and clinical sciences has improved our understanding of MTB transmission that, until recently, had remained largely unchanged for over 50 years. Figure 1 illustrates the MTB transmission cycle. Interrupting any process in MTB's natural history will reduce rates of transmission at a population level.

Aerosol generation and inhalation: Individuals with pulmonary TB aerosolise MTB, placing their contacts at risk of infection (Figure 1). This occurs at a faster rate during coughing. ${ }^{27}$ There is some evidence that speech $^{28}$ and singing ${ }^{29}$ are effective aerosol generating activities, though these studies focussed only on droplets originating in the mouth. Whilst the largest respiratory droplets fall to the ground, rapid evaporation means many droplets attain a sufficiently low mass before settling that they remain suspended in air currents until either inhaled or ventilated out of the room. ${ }^{30}$ Important new insights into MTB transmission have come from 'cough box' experiments. ${ }^{31}$ Here, TB patients were asked to cough 'as frequently as was comfortable' for five minutes into a 'cough aerosol sampling system'. Whilst this may not be physiological, these experiments suggest that most MTB that is aerosolised during coughing is in droplets small enough, even without evaporation, to remain suspended in the air. ${ }^{31}$ These cough box experiments ${ }^{31}, 32$, consistent with studies from guinea pig facilities ${ }^{33,34}$ and molecular epidemiological observations ${ }^{35-37}$, suggest that some people with TB may be much more infectious than others. Early animal 
experiments demonstrated that MTB in smaller droplets more readily produced tubercles in the lung than did MTB in larger droplets, presumably as they escape filtration in the upper airways. ${ }^{38}$ The use of surgical masks by TB patients has been shown to reduce transmission to guinea pigs by $56 \%$, suggesting they partially block aerosolisation of the relevant respiratory droplets. ${ }^{39}$

Establishing infection or disease: The quantity and characteristics of the inhaled droplet predict clinical outcomes, with early experiments demonstrating that infectious dose predicts risk of progression to disease. ${ }^{40}$ In the cough box experiments, the quantity of aerosolised MTB produced by individuals predicted infection in household contacts better than smear grade or time to culture positivity. ${ }^{32}$ It has recently been proposed that larger droplets, settling in the upper airway, might result in immune memory and a positive test for infection but little risk of progression to disease. ${ }^{40}$ That some highly exposed individuals fail to become TST or IGRA positive and the recent discovery of genetic loci that predict TST positivity suggest that it is possible to clear MTB infection without developing an adaptive immune response - so called 'early clearance' ${ }^{41}$ This process is probably important epidemiologically but, given it leaves no footprint, is difficult to study. There is a growing appreciation, informed by animal studies and advanced imaging techniques, that a binary classification of TB into latent infection and active disease may be too simplistic. ${ }^{42}{ }^{43}$ Some individuals with positive tests for infection may have cleared the organism, ${ }^{43}$ and periods of active replication in 'latent $\mathrm{TB}^{\prime}$ ' have been observed. ${ }^{42}$ Pulmonary MTB infection towards the more active end of the infection-to-disease spectrum is probably necessary for infectiousness.

Duration of infectiousness: There is a widely-held view that the infectiousness of patients diminishes sufficiently after two weeks of anti-tuberculous therapy such that transmission to contacts is unlikely. ${ }^{44}$ Many guidelines rely on proxy measures of infectiousness, e.g. smear status or culture conversion times. It is worth noting that median time to culture conversion amongst patients treated under daily direct observation for drug-susceptible TB in Peru was 37 days. ${ }^{45}$ However, in patients on treatment, the association between sputum smear or culture status and infectiousness is not straightforward, ${ }^{46-50}$ and is likely to be influenced not only by organism viability in respiratory secretions but also by the capacity to generate aerosolised MTB through coughing. ${ }^{27}$ Since cough frequency diminishes on treatment, ${ }^{51}$ assumptions about infectiousness based upon culture conversion times may overestimate risk. Furthermore, organisms that propagate in culture may not thrive when exposed to a hostile immune system in the alveoli.

Infectiousness can be studied in guinea pig facilities in which the number of animals infected following exposure to air exhausted from isolation rooms containing TB patients is measured. Such experiments show that effective therapy is associated with markedly fewer MTB infections than are seen before treatment is initiated or when isolates are not fully susceptible to the treatment regimen. ${ }^{34}$, 50 However, the guinea pigs in these experiments remained exposed to the patients for many weeks. Thus, these experiments have not yet reliably established a time window after which a patient can be considered to be no longer infectious. Most, if not all, household contacts are more likely to be infected by an index patient in the pre-treatment period than once treatment is initiated. ${ }^{52}$ This is due to both the likely longer duration of exposure and to greater infectiousness pre-treatment. This may also hold for patients with drug-resistant $\mathrm{TB}$, amongst whom culture conversion times will typically be longer, particularly if there is delay in initiating effective treatment.

\section{Measuring transmission in populations}

Even in the highest burden communities, the prevalence and annual incidence of active TB disease rarely exceed $2 \%$. This, in addition to incomplete surveillance data, particularly in high burden settings, and tests for MTB infection with poor sensitivity and specificity, make the measurement of MTB transmission in populations a challenge and the routine determination of incident TB disease unfeasible. ${ }^{53}$ Measuring 
transmission therefore relies on proxy measures, assumptions and combination approaches. There is debate about the best approach to measuring MTB transmission in trials of control interventions. ${ }^{54-56}$ Shortterm reductions in disease prevalence, for example, are difficult to interpret as prevalence is influenced not only by transmission, but also by progression from infection to disease and disease duration. ${ }^{54}$ The common tests for MTB infection are summarised in Panel 1. Approaches to measuring MTB transmission in populations are summarised in Table 1.

Traditional approaches: Tuberculin surveys in school children are a classical means of estimating MTB transmission at a population level. While age assortative mixing may mean paediatric infections do not fully reflect MTB transmission between adults 54,57,66, repeated TST surveys might still allow estimates of the trend in force of infection over time to be attained. Trend estimates based on tuberculin surveys are fairly robust and not greatly influenced by the proportion of children with BCG scars or the cut point used to define a 'positive' test. ${ }^{65,67}$

Molecular approaches: Tools for strain typing MTB include spoligotyping, which has limited resolution; Restriction Fragment Length Polymorphism (RFLP) ${ }^{68}$ and Mycobacterial Interspersed Repetitive Units Variable Number of Tandem Repeats (MIRU-VNTR), ${ }^{69,70}$ both of which have been widely used; and Whole Genome Sequencing (WGS). These molecular epidemiological tools provide evidence for or against potential linkages between two or more cases of active TB and have led to a number of critical insights into MTB transmission. ${ }^{71}$ As these techniques require a bacterial isolate, molecular epidemiology, with rare exceptions, ${ }^{72}$ only captures infections that have progressed to active TB disease. ${ }^{73}$ Molecular epidemiology cannot distinguish changes in transmission intensity from changes in the rate of progression to active TB shortly after infection resulting, for example, from varying levels of immunosuppression. The better resolution of WGS and steep reductions in cost mean it is likely to eventually replace existing strain typing techniques. However, molecular epidemiology using WGS will require an understanding of the rate at which mutations occur. Recent studies suggest that, in active disease, single nucleotide polymorphisms (SNPs) emerge, on average, at half a SNP per genome per year or slower. ${ }^{37,74-76}$ Most of the patients in these studies were on TB treatment and there was considerable variation in the rate at which mutations occurred. Primate studies suggest a similar mutation rate and that the mutation rate may not differ substantially between active and latent infection. ${ }^{77}$ However, limited data suggest that, in man, mutations accumulate more slowly during latent infection. ${ }^{78}$ Occasional accelerated intra-patient 'microevolution' events, ${ }^{79}$ and the slow rate at which SNPs accumulate may make inferring chains of transmission from MTB genotypes alone challenging. Probabilistic models that also incorporate epidemiological and clinical data may be needed. ${ }^{80}$ With molecular epidemiology using WGS, as with older strain typing techniques, adequate study duration, a high sampling fraction and careful documentation, follow up and reporting are important. ${ }^{16,81-83}$ However, novel approaches to the analysis of sequence data may allow population level inferences to be made from smaller samples.

Air sampling: There is interest in attempting to directly detect aerosolised MTB from room air. This might allow quantification of MTB exposure in putative sites of transmission. A few demonstration studies, using polymerase chain reaction (PCR) on room air filtrate, suggest this may be feasible. ${ }^{84-86}$ Whilst PCR detection of MTB DNA does not necessarily mean organisms are viable, it suggests that individual(s) with pulmonary TB have produced bioaerosols in the space. This should be, at least in theory, a reasonable proxy for transmission risk.

\section{The Wells-Riley Equation}

Room ventilation and social contact patterns determine whether others are exposed to MTB that has been aerosolised. The Wells-Riley Equation ${ }^{87}$ is used to model the transmission of respiratory pathogens, such as MTB, that are spread by crowd rather than close contact. Transmission risk in a defined space over time $t$ is modelled as a Poisson process. 


\section{Probability of transmission $=1-e^{-I q p t / Q}$}

$I$ is the number of infectious individuals present

$q$ is the rate at which infectious individuals produce infectious 'quanta'

$p$ is the rate at which susceptible individuals breathe

and $Q$ is the rate at which air from the space is exchanged with uncontaminated air (ventilation)

Riley and colleagues defined 'quanta' as 'the number of infectious airborne particles required to infect which may be one or more airborne particles'. ${ }^{87}$ This value is often assumed or fitted to data. Various attempts have been made to empirically estimate $q$ for TB by venting air exhaled by TB patients over experimental animals. Two sets of experiments in the pre-HIV era estimated quanta production at 0.62$0.82^{88}$ and $1.25^{89}$ per hour using a heterogeneous group of TB patients. ${ }^{39},{ }^{90}$ More recently, Escombe et al. obtained an estimate of 8.2 quanta per hour in a group of HIV positive patients in Lima, Peru. ${ }^{34}$ These data disguise huge heterogeneity in infectiousness with the most infectious patients in each study producing 60 and 226 infectious quanta per hour respectively. High rates of quanta production have been measured in patients with advanced multidrug-resistant TB (MDR-TB), ${ }^{39}$ and very high rates estimated in outbreaks related to aerosol generating procedures. ${ }^{91}$ Interestingly, estimates of $q$ obtained by fitting to data from high burden communities are lower than those obtained empirically. ${ }^{92}$ This may be because untreated patients in the community are at an earlier stage in their illness, and hence less infectious than the diagnosed patients used in the animal studies.

The Wells-Riley Equation has a number of important limitations. It assumes that air in the space is fully mixed, and does not account for heterogeneity in infectiousness or susceptibility to infection. Adaptations to the equation have been published. One widely used variant uses instead a 'rebreathed fraction' obtained from paired indoor and outdoor carbon dioxide $\left(\mathrm{CO}_{2}\right)$ measurements. ${ }^{93}$ This avoids the need to measure $Q$, which can be technically challenging. In many settings, there are substantial spatial and temporal variations in $\mathrm{CO}_{2}$ concentrations. Not obtaining contemporaneous $\mathrm{CO}_{2}$ measurements from directly outside the buildings studied may be reasonable where strong winds limit local spatial and temporal variations in $\mathrm{CO}_{2}{ }^{92}, 94$ but would not be reasonable in other circumstances. A number of important insights have been derived using the Wells-Riley approach. For example, one study suggested that active case finding could not control high levels of MTB transmission in a South African prison if levels of overcrowding and poor ventilation were not also addressed..$^{95}$ Another South African paper, which used the equation to predict settings in which MTB transmission might occur, is described below. ${ }^{92}$

\section{Research Priorities}

There is much left to be learned about MTB transmission. Here, we outline priority areas for future research. Approaches to interrupting MTB transmission include active case finding, the provision of isoniazid preventive therapy (IPT) and TB infection control. There have recently been large trials published of active case finding and mass IPT to interrupt MTB transmission. The ZAMSTAR result may be the first empirical data suggesting that active screening for TB disease impacts on TB transmission at a populationlevel.55, 96 The Thibela TB trial, conducted in a setting with a very high force of infection, found mass administration of IPT protected individuals whilst on treatment but had no impact on TB incidence in the wider community. ${ }^{20,22}$

Infection control: There has been much less research on TB infection control, which is conventionally described in three domains - administrative controls (which aim to minimise contamination of shared air by infectious subjects e.g. cough triage, early diagnosis and treatment), environmental controls (which aim 
to minimise exposure through removal of contaminated air) and personal protection measures (which aim to minimise inhalation of contaminated air e.g. N95 respirators). ${ }^{97}$ A review of observational and animal studies concluded that there is strong evidence supporting the role of ventilation as an environmental control in reducing the risk of airborne transmission of MTB. ${ }^{98}$ Of the many ways of increasing ventilation, increased mechanical ventilation, ${ }^{99}$ natural ventilation through increased window-opening, ${ }^{100}$ and winddriven roof turbines have been considered specifically as means to reduce MTB infection risk. ${ }^{101}$ Natural ventilation has been recommended by the WHO as an effective way to reduce infection. ${ }^{97}$ Air disinfection tools, particularly upper room Ultra Violet Germicidal Irradiation (UVGI), have also been studied with UVGI leading to steep reductions in MTB transmission from TB patients to experimental animals ${ }^{102,103}$

Implementing environmental controls is not always straightforward. An increase in indoor levels of outdoor pollution, security concerns, exposure to outdoor hazards such disease vectors, a loss of thermal comfort, energy loss through the exfiltration of conditioned (heated or cooled) indoor air, and higher running and maintenance costs of mechanical systems are side-effects of increased ventilation, ${ }^{104}$ and may make such measures unacceptable to occupants. Therefore, the ideal retrofit and design measures employed in a building should account for occupant patterns, numbers, and preferences, the climate and surrounding environment, building geometry, and the materials and budget available. Building simulation tools may be employed to predict the optimal design or retrofit of buildings to maximise ventilation according to specified criteria. ${ }^{105,106}$

The FAST approach to TB infection control in congregate settings has recently been promoted and advocates Finding TB cases Actively, Separating safely, and Treating effectively. ${ }^{107}$ A trial of the FAST approach is about to commence in Peru (NCT 02355223) with TST conversion among healthcare workers as an endpoint. However, the lack of a comparator group may limit the strength of the conclusions that can be reached. While a relationship between ventilation rate and TB transmission is clear, there is little empirical evidence on the effectiveness of infection control interventions in reducing transmission, with the majority of studies using animal surrogates or ventilation measurements as a proxy for transmission risk. A notable exception was the Tuberculosis Ultraviolet Shelter Study, which showed that environmental modifications can be safely implemented at scale. ${ }^{108}$ Whilst there were too few TST conversions among residents of the shelters to demonstrate an impact on MTB transmission, similar studies in higher burden settings would be valuable in quantifying the impact of $\mathrm{TB}$ infection control interventions on transmission to human occupants and, potentially, on transmission in the surrounding community. ${ }^{109}$ An important knowledge gap is whether domestic infection control implemented at diagnosis can mitigate against secondary infections in patients managed in the community. To our knowledge, there have been no trials of such interventions. This is an important question, perhaps in MDR-TB and certainly in extensively drugresistant TB (XDR-TB), where chemotherapy may not promptly reduce infectiousness and where the consequences of transmission may be severe.

Locating MTB transmission: Historically, households have been considered a major focus of MTB transmission. However, three molecular epidemiology studies from Sub Saharan Africa, ${ }^{17,110,111}$ and one from rural Vietnam, ${ }^{112}$ all suggest that most transmission occurs between rather than within households. In these high burden settings, this likely reflects a high transmission risk outside the home rather than a reduction in the risk of transmission to household contacts. Other evidence also suggests that, overall, most transmission may occur outside the household, ${ }^{113,114}$ but studies suggest this is age dependent with young children more likely to have been infected by a household member. ${ }^{115-117}$ It is likely that transmission in indoor congregate settings is important in high burden settings. For example, time working in public transport is strongly associated with TST positivity in Lima, Peru. ${ }^{118}$ Understanding which settings are 
important should be a research priority, as this would allow infection control and active case finding interventions to be better targeted. ${ }^{109}$

The Wells-Riley Equation and its variants have been used to estimate MTB transmission risk by location. Studies adopting these approaches have estimated ventilation or likely exposure to exhaled bioaerosols based on $\mathrm{CO}_{2}$ levels. These methods have recently been applied to study transmission in a Cape Town township. Data were collected on $\mathrm{CO}_{2}$ concentrations in multiple settings visited by 63 adolescents. It was estimated that $93 \%$ of total exposure to 'rebreathed air' occurred in a few locations: own home, visited homes, public transport, work or school. ${ }^{94}$ The same research group used a modified Wells-Riley Equation, $\mathrm{CO}_{2}$ measurements and social contact pattern data to estimate the proportion of overall MTB transmission by location. They found that, in the same Cape Town township, 50\% of incident infections among 15-19 year olds may take place in school, that workplaces are important places for adult transmission and that household and public transport may be important sites of transmission between age groups. ${ }^{92}$ These inferences are potentially useful but the studies were small. The conclusions may be context-specific and assume TB disease prevalence is the same in each location within an age and sex group, which is probably not the case. Similar studies need to be undertaken on larger scales and in more settings, ideally combined with location specific estimates of TB prevalence.

Health facilities, particularly in HIV endemic areas, are an important setting in which infectious TB patients mix with susceptible persons. TB patients attend healthcare facilities prior to diagnosis, when presenting with TB symptoms, and during the course of TB treatment. Delays in recognition, diagnosis and isolation of infectious cases augment the risk of nosocomial transmission from unsuspected index cases. Overcrowded outpatient clinics and Emergency Departments congregate vulnerable subjects in settings where the likelihood of exposure to patients with infectious TB is relatively high. In one Emergency Department study in Lima, Peru, IGRA conversion was observed in 30\% of healthcare workers during a 12 month period compared to $0 \%$ of hospital security and domestic personnel; ${ }^{119}$ remarkably one third of patients identified with TB in the study were attending the hospital for an apparently unrelated reason (trauma, pregnancy etc.) highlighting the importance of the unrecognized risk. Nosocomial transmission played an important role in the Tugela Ferry extensively drug resistant tuberculosis XDR-TB outbreak. ${ }^{120}$ Future research should attempt to quantify the proportion of MTB transmission in high burden settings that occurs within healthcare facilities, as well as the impact of programmatic infection control interventions on this proportion.

Spatial heterogeneity in the incidence of $\mathrm{TB}$ and drug-resistant $\mathrm{TB}$ is evident in analyses of programmatically collected data and has been well documented in many studies, ${ }^{121-123}$ raising the possibility that targeted interventions may be effective. At this time, however, the mechanisms driving such heterogeneity are not completely understood; for example, localized transmission and or aggregation of individuals sharing risk factors for infection or progression may combine to generate such patterns of disease. A better understanding of this spatial heterogeneity might usefully inform targeted TB control interventions. The spatial extent of TB transmission networks in these settings is not known. Better data on the geographical extent of social contacts relevant for TB transmission will assist in designing intervention studies and it would be helpful to see research addressing this question.

Drug resistance and transmissibility: Globally, there were an estimated 480,000 incident cases of MDR-TB in 2013. The proportion of new cases that are infected with MDR-TB is around 3.5\% and this has not changed appreciably over the period 2008-13. ${ }^{3}$ MDR-TB is disproportionately distributed, with the highest rates seen in Central Asia and Eastern Europe where, in several countries, a high proportion of MDR-TB cases have no previous history of TB treatment. This suggests high levels of MDR-TB transmission. 
Projections of the future burden of MDR-TB depend critically on estimates of the reproductive potential of drug resistant strains as compared to drug sensitive strains. ${ }^{124,125}$ This reproductive potential, which can be quantified as the expected number of secondary infectious cases attributable to a single infectious case, is the product of several factors: the duration of infectiousness, the rate at which respiratory exposures occur, the probability that exposure results in transmission, and the probability that infection progresses to infectious active disease. ${ }^{126}$ Drug-resistance may affect several of these factors. For example, the duration of infectiousness is often longer in individuals with MDR-TB as delays in the diagnosis of resistance can lead to delayed initiation of effective treatment. However, the probability of establishing a successful infection or of infection leading to disease will be decreased if resistance-conferring mutations have a fitness cost. ${ }^{127}$ In vitro experiments (e.g. competitive growth assays which may measure biological fitness) and observational studies (e.g. contact tracing and molecular clustering studies which measure effects of both biological fitness and differences in duration of infectiousness) suggest a wide variety of possible overall relationships between drug-resistance and transmission. ${ }^{128}$ Furthermore, even where there are clear biological costs associated with resistance, these may be ameliorated by compensatory mutations. ${ }^{129}, 130$ WGS analyses of clinical strains suggest successful transmission of MDR strains in disparate settings such as South Africa ${ }^{131}$ and Russia ${ }^{132}$. However, a recent household contact study suggested circulating MDR-TB strains in Peru were less likely to result in disease in household contacts than drug sensitive strains. ${ }^{133}$ The data on XDR transmission, at least during the time of observation, are also mixed. ${ }^{131,134}$ Were the fitness of drug resistant strains to increase over time, these mixed results might reflect differences in the maturity of drug resistant TB epidemics. ${ }^{135}$ Given the importance of reproductive potential to projections of the MDR and XDR-TB epidemics, this remains a research priority.

Antiretroviral therapy and MTB transmission: Our understanding of the effects of HIV on MTB transmission remains limited. ${ }^{73}$ Some data suggest that HIV positive people with TB disease make a small contribution to MTB transmission. The arrival of HIV in Tanzania was associated with an increase in TB incidence but a decline in annual risk of TB infection measured in a series of tuberculin surveys. ${ }^{67}$ The arrival of HIV in South Africa was associated with a marked increase in TB incidence in HIV positive but not HIV negative miners. ${ }^{136}$ This finding was replicated in a prospective cohort study in business employees in Harare. ${ }^{137}$ These studies were conducted before antiretroviral therapy was widely available. Household contact studies suggest transmission to household contacts is lower when the index case has more advanced HIVrelated immunosuppression. ${ }^{138}$ Molecular epidemiology suggests that, in a South African township, HIV negative people are more likely to be the index cases in strain clusters than HIV positive people. ${ }^{17,}{ }^{139}$ However, a study in Malawi using WGS found no association between HIV status or receipt of ART and the probability of being linked to secondary cases. ${ }^{76}$ There are several potential explanations for these observations. HIV positive people are more likely to have smear-negative or extrapulmonary disease, which are less infectious. Shorter disease duration ${ }^{140}$ due to faster progression to death or treatment may limit opportunity to transmit, as might reduced social contact as a result of greater morbidity. However, whilst people in HIV care may have their TB diagnosed faster, healthcare facilities may be important sites of transmission.

Following publication of the START ${ }^{141}$ and ANRS TEMPRANO ${ }^{142}$ trials, the WHO guidelines have recently been updated. ${ }^{143}$ It is now recommended that ART be provided to all people living with HIV irrespective of CD4 cell count. In response, national policies are likely to be updated to recommend earlier initiation of ART. ART reduces TB disease incidence rates in HIV cohorts by approximately two-thirds. ${ }^{144-146}$ Short-term reductions in population level TB disease rates have also been observed in communities in South Africa and Malawi where ART has been scaled up rapidly. ${ }^{147,}{ }^{148}$ This may be largely explained by reduced progression from infection to disease rather than by reductions in transmission. The longer-term impact of ART on population-level TB disease burden and the impact on MTB infection incidence are uncertain. ART 
certainly affects longevity, levels of contact with healthcare services and susceptibility to TB disease. It may also affect TB disease duration and phenotype, including the presence of cavities, smear positivity, ${ }^{149,150}$ and the relative frequency of extra-pulmonary disease, all of which might affect infectiousness. Furthermore, reduced morbidity as a result of ART might result in increased levels of social contact.

An influential modelling study estimating the impact of the roll-out of annual HIV testing and immediate ART on TB disease incidence in nine African countries predicted a $21 \%$ (range: $10 \%-31 \%$ ) reduction in the cumulative AIDS-related TB disease incidence over the first five years, and a $48 \%$ (range: 37\%-55\%) reduction in the incidence of TB disease at five years. ${ }^{151}$ A multi-model analysis for the time period 20142033, estimated that increasing ART coverage to $80 \%$ of those with CD4 count $<350$ cells $/ \mathrm{mm}^{3}$ could reduce TB incidence by $8-14 \%$ and, if ART were provided to all HIV infected individuals (at current levels of access), incidence could be reduced by $6-30 \% .{ }^{152}$ However, a more recent modelling study suggested that the longer-term impact of expanded ART access was less certain. ${ }^{153}$ Whilst TB incidence should initially decline, the model predicted that, if good adherence and immunologic responses to ART are not sustained and combined with effective HIV preventive interventions, increases in TB disease incidence might occur despite high levels of ART coverage. ${ }^{153}$ Following publication of the new WHO guidelines, ${ }^{143}$ it is likely that more people will be initiated on ART at higher CD4 counts. Given the substantial impact - either positive or negative - that this may have on TB in HIV endemic settings, the impact of HIV and ART on transmission dynamics should be a focus of research.

\section{Conclusions}

Addressing MTB transmission is critical to achieving TB control in high burden settings. Repeated surveys measuring TB infection in the same community, including young adults, offer a feasible measure of TB transmission. This approach might be used in trials in high burden settings to enable the impact of interventions on transmission to be disaggregated from their effects on rates of progression to disease or disease duration. The coming years will likely see innovative and exciting research on MTB transmission in high burden settings. When designing studies, researchers should be mindful of the context in such settings. Priority should be given to the development and evaluation of TB control strategies that place minimal additional demands on poor patients and overstretched healthcare systems, ${ }^{109}$ and or that involve elements of social protection or health system strengthening.

\section{SEARCH STRATEGY}

To find articles pertinent to this narrative review, we searched PubMed to 1 October 2015. We sought articles published in English using the terms 'tuberculosis' and 'transmission'. We also included papers included in reference lists or suggested by members of the review team.

\section{AUTHOR CONTRIBUTIONS}

TAY, PYK, GMK, JGT, RGW, TC, FGC, DAJM and IA drafted sections of the manuscript. All authors commented on and edited the manuscript. TAY, PYK and IA prepared the final draft. All authors approved the final version of the manuscript prior to submission.

\section{FUNDING}

The M.tb Transmission meeting from which this article stemmed was funded by the TB Modelling and Analysis Consortium (OPP1084276), UCL Population Health Domain and the TB Centre at the London 
School of Hygiene and Tropical Medicine. TAY is funded via a studentship from the Medical Research Council (UK). PYK is funded via Wellcome Trust clinical research training fellowship (100137/Z/12/Z). RGW is funded by the Medical Research Council (UK) (MR/J005088/1), the Bill and Melinda Gates Foundation (TB Modelling and Analysis Consortium: OPP1084276), CDC/PEPFAR via the Aurum Institute (U2GPS0008111), and USAID/IUTLD/The Union North America (TREAT TB: Technology, Research, Education, and Technical Assistance for Tuberculosis; GHN-A-OO-08-00004-00). TC is funded via a NIH Research Project Grant (R01 AI112438-01). IA is funded by the UK Medical Research Council, National Institute for Health Research and Public Health England.

\section{ROLE OF THE FUNDING SOURCE}

Our funders had no involvement in the drafting of or decision to submit this manuscript.

\section{ETHICS APPROVAL}

Not applicable.

\section{REFERENCES}

1. Glaziou P, Falzon D, Floyd K, Raviglione M. Global epidemiology of tuberculosis. Semin. Respir. Crit. Care Med. 2013; 34(1): 3-16.

2. WHO. End TB Strategy. Draft global strategy and targets for tuberculosis prevention, care and control after 2015. Documentation for World Health Assembly 67.

http://apps.who.int/gb/ebwha/pdf files/WHA67/A67 11-en.pdf. Geneva: World Health Organisation; 2014 [Accessed on 22/3/2015].

3. WHO. Global tuberculosis control: WHO report 2014.

http://apps.who.int/iris/bitstream/10665/137094/1/9789241564809 eng.pdf. Geneva: World Health Organisation; 2014 [Accessed on 22/3/2015].

4. Dowdy DW, Chaisson RE. The persistence of tuberculosis in the age of DOTS: reassessing the effect of case detection. Bull. World Health Organ. 2009; 87(4): 296-304.

5. WHO. STOP TB Strategy. Building on and enhancing DOTS to meet the TB-related Millennium Development Goals. http://whqlibdoc.who.int/hq/2006/WHO HTM STB 2006.368 eng.pdf Geneva: World Health Organisation; 2006 [Accessed on 23/3/2015].

6. Dodd PJ, Gardiner E, Coghlan R, Seddon JA. Burden of childhood tuberculosis in 22 high-burden countries: a mathematical modelling study. Lancet Glob Health 2014; 2(8): e453-9.

7. Jenkins HE, Tolman AW, Yuen CM, et al. Incidence of multidrug-resistant tuberculosis disease in children: systematic review and global estimates. Lancet 2014; 383(9928): 1572-9.

8. Gopi PG, Subramani R, Nataraj T, Narayanan PR. Impact of BCG vaccination on tuberculin surveys to estimate the annual risk of tuberculosis infection in south India. Indian J. Med. Res. 2006; 124(1): 71-6.

9. Munim A, Rajab Y, Barker A, Daniel M, Williams B. Risk of Mycobacterium tuberculosis infection in Somalia: national tuberculin survey 2006. East Mediterr Health J 2008; 14(3): 518-30.

10. Doocy SC, Todd CS, Llainez YB, Ahmadzai A, Burnham GM. Population-based tuberculin skin testing and prevalence of tuberculosis infection in Afghanistan. World Health Popul 2008; 10(1): 4453.

11. Addo KK, van den Hof S, Mensah GI, et al. A tuberculin skin test survey among Ghanaian school children. BMC Public Health 2010; 10: 35.

12. Hoa NB, Cobelens FG, Sy DN, Nhung NV, Borgdorff MW, Tiemersma EW. First national tuberculin survey in Viet Nam: characteristics and association with tuberculosis prevalence. Int. J. Tuberc. Lung Dis. 2013; 17(6): 738-44.

13. Kritzinger FE, den Boon S, Verver S, et al. No decrease in annual risk of tuberculosis infection in endemic area in Cape Town, South Africa. Trop. Med. Int. Health 2009; 14(2): 136-42. 
14. Wood R, Lawn SD, Johnstone-Robertson S, Bekker LG. Tuberculosis control has failed in South Africa - time to reappraise strategy. S. Afr. Med. J. 2011; 101(2): 111-4.

15. Andrews JR, Hatherill M, Mahomed H, et al. The Dynamics of QuantiFERON(R)-TB Gold In-Tube Conversion and Reversion in a Cohort of South African Adolescents. Am. J. Respir. Crit. Care Med. 2015. 16. Houben RM, Glynn JR. A systematic review and meta-analysis of molecular epidemiological studies of tuberculosis: development of a new tool to aid interpretation. Trop. Med. Int. Health 2009; 14(8): 892-909.

17. Middelkoop K, Mathema B, Myer L, et al. Transmission of Tuberculosis in a South African Community With a High Prevalence of HIV Infection. J. Infect. Dis. 2015; 211(1): 53-61.

18. Houben RM, Crampin AC, Ndhlovu R, et al. Human immunodeficiency virus associated tuberculosis more often due to recent infection than reactivation of latent infection. Int. J. Tuberc. Lung Dis. 2011; 15(1): 24-31.

19. Samandari T, Agizew TB, Nyirenda S, et al. 6-month versus 36-month isoniazid preventive treatment for tuberculosis in adults with HIV infection in Botswana: a randomised, double-blind, placebo-controlled trial. Lancet 2011; 377(9777): 1588-98.

20. Churchyard GJ, Fielding KL, Lewis JJ, et al. A trial of mass isoniazid preventive therapy for tuberculosis control. N. Engl. J. Med. 2014; 370(4): 301-10.

21. Houben RM, Sumner T, Grant AD, White RG. Ability of preventive therapy to cure latent Mycobacterium tuberculosis infection in HIV-infected individuals in high-burden settings. Proc. Natl. Acad. Sci. U. S. A. 2014; 111(14): 5325-30.

22. Vynnycky E, Sumner T, Fielding KL, et al. Tuberculosis Control in South African Gold Mines: Mathematical Modeling of a Trial of Community-Wide Isoniazid Preventive Therapy. Am. J. Epidemiol. 2015.

23. Hawn TR, Day TA, Scriba TJ, et al. Tuberculosis vaccines and prevention of infection. Microbiol. Mol. Biol. Rev. 2014; 78(4): 650-71.

24. Cohen T, van Helden PD, Wilson D, et al. Mixed-strain mycobacterium tuberculosis infections and the implications for tuberculosis treatment and control. Clin. Microbiol. Rev. 2012; 25(4): 708-19. 25. Getahun H, Matteelli A, Abubakar I, et al. Management of latent Mycobacterium tuberculosis infection: WHO guidelines for low tuberculosis burden countries. Eur. Respir. J. 2015.

26. Rieder HL. Epidemiological basis of tuberculosis control. Paris: International Union Against Tuberculosis and Lung Disease; 1999.

27. Turner RD, Bothamley GH. Cough and the Transmission of Tuberculosis. J. Infect. Dis. 2014.

28. Loudon RG, Roberts RM. Droplet expulsion from the respiratory tract. Am. Rev. Respir. Dis. 1967; 95(3): 435-42.

29. Loudon RG, Roberts RM. Singing and the dissemination of tuberculosis. Am. Rev. Respir. Dis. 1968; 98(2): 297-300.

30. Wells WF. On air-bourne infection. Study II. Droplets and droplet nuclei. American journal of hygiene 1934; 20(3): 611-8.

31. Fennelly KP, Jones-Lopez EC, Ayakaka I, et al. Variability of infectious aerosols produced during coughing by patients with pulmonary tuberculosis. Am. J. Respir. Crit. Care Med. 2012; 186(5): 450-7.

32. Jones-Lopez EC, Namugga O, Mumbowa F, et al. Cough aerosols of Mycobacterium tuberculosis predict new infection: a household contact study. Am. J. Respir. Crit. Care Med. 2013; 187(9): 1007-15. 33. Sultan L, Nyka W, Mills C, O'Grady F, Wells W, Riley RL. Tuberculosis disseminators. A study of the variability of aerial infectivity of tuberculous patients. Am. Rev. Respir. Dis. 1960; 82: 358-69.

34. Escombe AR, Moore DA, Gilman RH, et al. The infectiousness of tuberculosis patients coinfected with HIV. PLoS Med. 2008; 5(9): e188.

35. Godfrey-Faussett P, Sonnenberg P, Shearer SC, et al. Tuberculosis control and molecular epidemiology in a South African gold-mining community. Lancet 2000; 356(9235): 1066-71.

36. Ypma RJ, Altes HK, van Soolingen D, Wallinga J, van Ballegooijen WM. A sign of superspreading in tuberculosis: highly skewed distribution of genotypic cluster sizes. Epidemiology 2013; 24(3): 395400.

37. Walker TM, Ip CL, Harrell RH, et al. Whole-genome sequencing to delineate Mycobacterium tuberculosis outbreaks: a retrospective observational study. Lancet Infect. Dis. 2013; 13(2): 137-46. 
38. Wells WF, Ratcliffe HL, Grumb C. On the mechanics of droplet nuclei infection; quantitative experimental air-borne tuberculosis in rabbits. Am J Hyg 1948; 47(1): 11-28.

39. Dharmadhikari AS, Mphahlele M, Stoltz A, et al. Surgical face masks worn by patients with multidrug-resistant tuberculosis: impact on infectivity of air on a hospital ward. Am. J. Respir. Crit. Care Med. 2012; 185(10): 1104-9.

40. Fennelly KP, Jones-Lopez EC. Quantity and Quality of Inhaled Dose Predicts Immunopathology in Tuberculosis. Front. Immunol. 2015; 6: 313.

41. Verrall AJ, Netea MG, Alisjahbana B, Hill PC, van Crevel R. Early clearance of Mycobacterium tuberculosis: a new frontier in prevention. Immunology 2014; 141(4): 506-13.

42. Barry CE, 3rd, Boshoff HI, Dartois V, et al. The spectrum of latent tuberculosis: rethinking the biology and intervention strategies. Nat Rev Microbiol 2009; 7(12): 845-55.

43. Esmail H, Barry CE, 3rd, Young DB, Wilkinson RJ. The ongoing challenge of latent tuberculosis. Philos. Trans. R. Soc. Lond. B Biol. Sci. 2014; 369(1645): 20130437.

44. National Institute for Health and Care Excellence. Tuberculosis: Clinical diagnosis and management of tuberculosis, and measures for its prevention and control (CG117). https://http://www.nice.org.uk/guidance/cg117/resources/guidance-tuberculosis-pdf: ; 2011.

45. Fitzwater SP, Caviedes L, Gilman RH, et al. Prolonged infectiousness of tuberculosis patients in a directly observed therapy short-course program with standardized therapy. Clin. Infect. Dis. 2010; 51(4): 371-8.

46. Kamat SR, Dawson JJ, Devadatta S, et al. A controlled study of the influence of segregation of tuberculous patients for one year on the attack rate of tuberculosis in a 5-year period in close family contacts in South India. Bull. World Health Organ. 1966; 34(4): 517-32.

47. Gunnels JJ, Bates JH, Swindoll H. Infectivity of sputum-positive tuberculous patients on chemotherapy. Am. Rev. Respir. Dis. 1974; 109(3): 323-30.

48. Menzies D. Effect of treatment on contagiousness of patients with active pulmonary tuberculosis. Infect. Control Hosp. Epidemiol. 1997; 18(8): 582-6.

49. Dharmadhikari AS, Nardell E. Serial acid fast bacilli smear and culture conversion rates over 26 weeks in a cohort of 93 sputum culture-positive tuberculosis (TB). Clin. Infect. Dis. 2011; 52(4): 554-6. 50. Dharmadhikari AS, Mphahlele M, Venter K, et al. Rapid impact of effective treatment on transmission of multidrug-resistant tuberculosis. Int. J. Tuberc. Lung Dis. 2014; 18(9): 1019-25.

51. Louden RG, Spohn SK. Cough frequency and infectivity in patients with pulmonary tuberculosis. Am. Rev. Respir. Dis. 1969; 99: 109-11.

52. Kasaie P, Andrews JR, Kelton WD, Dowdy DW. Timing of tuberculosis transmission and the impact of household contact tracing. An agent-based simulation model. Am. J. Respir. Crit. Care Med. 2014; 189(7): 845-52.

53. Dye C, Bassili A, Bierrenbach AL, et al. Measuring tuberculosis burden, trends, and the impact of control programmes. Lancet Infect. Dis. 2008; 8(4): 233-43.

54. Cobelens F, van Leth F, van 't Hoog A. Design of pragmatic trials of tuberculosis interventions. Lancet 2014; 383(9913): 213-4.

55. Ayles H, Muyoyeta M, Du Toit E, et al. Effect of household and community interventions on the burden of tuberculosis in southern Africa: the ZAMSTAR community-randomised trial. Lancet 2013; 382(9899): 1183-94.

56. Ayles H, Floyd S, Beyers N, Godfrey-Faussett P, team Z. Design of pragmatic trials of tuberculosis interventions - authors' reply. Lancet 2014; 383(9913): 214-5.

57. Rieder H. Annual risk of infection with Mycobacterium tuberculosis. Eur. Respir. J. 2005; 25(1): 181-5.

58. Neuenschwander BF. Bayesian Mixture Analysis for Tuberculin Induration Data. http://www.tbrieder.org/research/mixture/mixture documentation.pdf: The Union; 2007 [Accessed on 26/3/2015].

59. Fine PE, Bruce J, Ponnighaus JM, Nkhosa P, Harawa A, Vynnycky E. Tuberculin sensitivity: conversions and reversions in a rural African population. Int. J. Tuberc. Lung Dis. 1999; 3(11): 962-75. 60. Aggerbeck H, Giemza R, Joshi P, et al. Randomised clinical trial investigating the specificity of a novel skin test (C-Tb) for diagnosis of M. tuberculosis infection. PLoS One 2013; 8(5): e64215. 
61. Berry MP, Graham CM, McNab FW, et al. An interferon-inducible neutrophil-driven blood transcriptional signature in human tuberculosis. Nature 2010; 466(7309): 973-7.

62. Cliff JM, Lee JS, Constantinou N, et al. Distinct phases of blood gene expression pattern through tuberculosis treatment reflect modulation of the humoral immune response. J. Infect. Dis. 2013; 207(1): 18-29.

63. Kaforou M, Wright VJ, Oni T, et al. Detection of tuberculosis in HIV-infected and -uninfected African adults using whole blood RNA expression signatures: a case-control study. PLoS Med. 2013; 10(10): e1001538.

64. van Leth F, van der Werf MJ, Borgdorff MW. Prevalence of tuberculous infection and incidence of tuberculosis: a re-assessment of the Styblo rule. Bull. World Health Organ. 2008; 86(1): 20-6.

65. Borgdorff M. Annual risk of infection- time for an update? Bull. World Health Organ. 2002; 80(6): 501-2.

66. Johnstone-Robertson SP, Mark D, Morrow C, et al. Social mixing patterns within a South African township community: implications for respiratory disease transmission and control. Am. J. Epidemiol. 2011; 174(11): 1246-55.

67. Egwaga SM, Cobelens FG, Muwinge H, Verhage C, Kalisvaart N, Borgdorff MW. The impact of the HIV epidemic on tuberculosis transmission in Tanzania. AIDS 2006; 20(6): 915-21.

68. van Embden JD, Cave MD, Crawford JT, et al. Strain identification of Mycobacterium tuberculosis by DNA fingerprinting: recommendations for a standardized methodology. J. Clin. Microbiol. 1993; 31(2): 406-9.

69. Supply P, Mazars E, Lesjean S, Vincent V, Gicquel B, Locht C. Variable human minisatellite-like regions in the Mycobacterium tuberculosis genome. Mol. Microbiol. 2000; 36(3): 762-71.

70. Supply P, Lesjean S, Savine E, Kremer K, van Soolingen D, Locht C. Automated high-throughput genotyping for study of global epidemiology of Mycobacterium tuberculosis based on mycobacterial interspersed repetitive units. J. Clin. Microbiol. 2001; 39(10): 3563-71.

71. Borgdorff MW, van Soolingen D. The re-emergence of tuberculosis: what have we learnt from molecular epidemiology? Clin. Microbiol. Infect. 2013; 19(10): 889-901.

72. du Plessis DG, Warren R, Richardson M, Joubert JJ, van Helden PD. Demonstration of reinfection and reactivation in HIV-negative autopsied cases of secondary tuberculosis: multilesional genotyping of Mycobacterium tuberculosis utilizing IS 6110 and other repetitive element-based DNA fingerprinting. Tuberculosis (Edinb) 2001; 81(3): 211-20.

73. Godfrey-Faussett P. Population-level control of HIV-related TB (oral presentation). 21st Conference of Retroviral and Opportunistic Infections (3-6 March 2014). Boston, Massachusetts. (http://www.croiwebcasts.org/console/player/22244?mediaType=audio\&) [Accessed on 27/3/2015].

74. Bryant JM, Schurch AC, van Deutekom H, et al. Inferring patient to patient transmission of Mycobacterium tuberculosis from whole genome sequencing data. BMC Infect. Dis. 2013; 13: 110. 75. Roetzer A, Diel R, Kohl TA, et al. Whole genome sequencing versus traditional genotyping for investigation of a Mycobacterium tuberculosis outbreak: a longitudinal molecular epidemiological study. PLoS Med. 2013; 10(2): e1001387.

76. Guerra-Assuncao J, Crampin A, Houben R, et al. Large-scale whole genome sequencing of provides insights into transmission in a high prevalence area. Elife 2015; 4.

77. Ford CB, Lin PL, Chase MR, et al. Use of whole genome sequencing to estimate the mutation rate of Mycobacterium tuberculosis during latent infection. Nat. Genet. 2011; 43(5): 482-6.

78. Colangeli R, Arcus VL, Cursons RT, et al. Whole genome sequencing of Mycobacterium tuberculosis reveals slow growth and low mutation rates during latent infections in humans. PLoS One 2014; 9(3): e91024.

79. Perez-Lago L, Comas I, Navarro Y, et al. Whole genome sequencing analysis of intrapatient microevolution in Mycobacterium tuberculosis: potential impact on the inference of tuberculosis transmission. J. Infect. Dis. 2014; 209(1): 98-108.

80. Didelot X, Gardy J, Colijn C. Bayesian inference of infectious disease transmission from wholegenome sequence data. Mol. Biol. Evol. 2014; 31(7): 1869-79. 
81. van Soolingen D, Borgdorff MW, de Haas PE, et al. Molecular epidemiology of tuberculosis in the Netherlands: a nationwide study from 1993 through 1997. J. Infect. Dis. 1999; 180(3): 726-36.

82. Murray M, Alland D. Methodological problems in the molecular epidemiology of tuberculosis. Am. J. Epidemiol. 2002; 155(6): 565-71.

83. Field N, Cohen T, Struelens MJ, et al. Strengthening the Reporting of Molecular Epidemiology for Infectious Diseases (STROME-ID): an extension of the STROBE statement. Lancet Infect. Dis. 2014; 14(4): 341-52.

84. Mastorides SM, Oehler RL, Greene JN, Sinnott JTt, Kranik M, Sandin RL. The detection of airborne Mycobacterium tuberculosis using micropore membrane air sampling and polymerase chain reaction. Chest 1999; 115(1): 19-25.

85. Wan GH, Lu SC, Tsai YH. Polymerase chain reaction used for the detection of airborne Mycobacterium tuberculosis in health care settings. Am. J. Infect. Control 2004; 32(1): 17-22.

86. Matuka O, Singh TS, Bryce E, et al. Pilot study to detect airborne Mycobacterium tuberculosis exposure in a South African public healthcare facility outpatient clinic. J. Hosp. Infect. 2015; 89(3): 192-6.

87. Riley EC, Murphy G, Riley RL. Airborne spread of measles in a suburban elementary school. Am. J. Epidemiol. 1978; 107(5): 421-32.

88. Riley RL, Mills CC, Nyka W, et al. Aerial dissemination of pulmonary tuberculosis: a two-year study of contagion in a tuberculosis ward. Am J Hyg 1959; 70(2).

89. Riley RL, Mills CC, O'Grady F, Sultan LU, Wittstadt F, Shivpuri DN. Infectiousness of air from a tuberculosis ward. Ultraviolet irradiation of infected air: comparative infectiousness of different patients. Am. Rev. Respir. Dis. 1962; 85: 511-25.

90. Riley RL, Nardell EA. Clearing the air. The theory and application of ultraviolet air disinfection. Am. Rev. Respir. Dis. 1989; 139(5): 1286-94.

91. Beggs CB, Noakes CJ, Sleigh PA, Fletcher LA, Siddiqi K. The transmission of tuberculosis in confined spaces: an analytical review of alternative epidemiological models. Int. J. Tuberc. Lung Dis. 2003; 7(11): 1015-26.

92. Andrews JR, Morrow C, Walensky RP, Wood R. Integrating social contact and environmental data in evaluating tuberculosis transmission in a South African township. J. Infect. Dis. 2014; 210(4): 597-603.

93. Rudnick SN, Milton DK. Risk of indoor airborne infection transmission estimated from carbon dioxide concentration. Indoor Air 2003; 13(3): 237-45.

94. Wood R, Morrow C, Ginsberg S, et al. Quantification of shared air: a social and environmental determinant of airborne disease transmission. PLoS One 2014; 9(9): e106622.

95. Johnstone-Robertson S, Lawn SD, Welte A, Bekker LG, Wood R. Tuberculosis in a South African prison - a transmission modelling analysis. S. Afr. Med. J. 2011; 101(11): 809-13.

96. Kranzer K, Afnan-Holmes H, Tomlin K, et al. The benefits to communities and individuals of screening for active tuberculosis disease: a systematic review. Int. J. Tuberc. Lung Dis. 2013; 17(4): 432-46.

97. WHO. WHO Policy on TB Infection Control in Health-Care Facilities, Congregate Settings and Households. http://whqlibdoc.who.int/publications/2009/9789241598323 eng.pdf Geneva: World Health Organisation; 2009 [Accessed on 26/3/2015].

98. Li Y, Leung GM, Tang JW, et al. Role of ventilation in airborne transmission of infectious agents in the built environment - a multidisciplinary systematic review. Indoor Air 2007; 17(1): 2-18.

99. Menzies D, Fanning A, Yuan L, FitzGerald JM. Hospital ventilation and risk for tuberculous infection in canadian health care workers. Canadian Collaborative Group in Nosocomial Transmission of TB. Ann. Intern. Med. 2000; 133(10): 779-89.

100. Escombe AR, Oeser CC, Gilman RH, et al. Natural ventilation for the prevention of airborne contagion. PLoS Med. 2007; 4(2): e68.

101. Cox H, Escombe R, McDermid C, et al. Wind-driven roof turbines: a novel way to improve ventilation for TB infection control in health facilities. PLoS One 2012; 7(1): e29589.

102. Escombe AR, Moore DA, Gilman RH, et al. Upper-room ultraviolet light and negative air ionization to prevent tuberculosis transmission. PLoS Med. 2009; 6(3): e43. 
103. Shenoi SV, Escombe AR, Friedland G. Transmission of drug-susceptible and drug-resistant tuberculosis and the critical importance of airborne infection control in the era of HIV infection and highly active antiretroviral therapy rollouts. Clin. Infect. Dis. 2010; 50 Suppl 3: S231-7.

104. WHO. WHO guidelines for indoor air quality: dampness and mould. http://www.euro.who.int/ data/assets/pdf file/0017/43325/E92645.pdf?ua=1 [Accessed on 26/3/2015]. Geneva: World Health Organisation; 2009 [Accessed on 26/3/2015].

105. Khan MAI, Noakes CJ, Toropov VV. Development of a numerical optimization approach to ventilation system design to control airborne contaminant dispersion and occupant comfort. Building Simulation 2012; 5(1): 39-50.

106. Kim SH, Augenbroe G. Decision support for choosing ventilation operations strategy in hospital isolation rooms: A multi-criterion assessment under uncertainty. Building and Environment 2013; 60: 305-18.

107. TB Care II. FAST: A Tuberculosis Infection Control Strategy. http://drtbnetwork.org/sites/default/files/FAST May 2013 Booklet.pdf: USAID; 2013 [Accessed on 25/1/2015].

108. Nardell EA, Bucher SJ, Brickner PW, et al. Safety of upper-room ultraviolet germicidal air disinfection for room occupants: results from the Tuberculosis Ultraviolet Shelter Study. Public Health Rep. 2008; 123(1): 52-60.

109. Yates TA, Tanser F, Abubakar I. Plan Beta for Tuberculosis: it's time to think seriously about poorly ventilated congregate settings. Int. J. Tuberc. Lung Dis. 2015; in press.

110. Verver S, Warren RM, Munch Z, et al. Proportion of tuberculosis transmission that takes place in households in a high-incidence area. Lancet 2004; 363(9404): 212-4.

111. Glynn JR, Guerra-Assuncao JA, Houben RM, et al. Whole Genome Sequencing Shows a Low Proportion of Tuberculosis Disease Is Attributable to Known Close Contacts in Rural Malawi. PLoS One 2015; 10(7): e0132840.

112. Buu TN, van Soolingen D, Huyen MN, et al. Tuberculosis acquired outside of households, rural Vietnam. Emerg. Infect. Dis. 2010; 16(9): 1466-8.

113. Narain R, Nair SS, Rao GR, Chandrasekhar P. Distribution of tuberculous infection and disease among households in a rural community. Bull. World Health Organ. 1966; 34(4): 639-54.

114. Brooks-Pollock E, Becerra MC, Goldstein E, Cohen T, Murray MB. Epidemiologic inference from the distribution of tuberculosis cases in households in Lima, Peru. J. Infect. Dis. 2011; 203(11): 1582-9. 115. Wood R, Johnstone-Robertson S, Uys P, et al. Tuberculosis transmission to young children in a South African community: modeling household and community infection risks. Clin. Infect. Dis. 2010; 51(4): 401-8.

116. Middelkoop K, Bekker LG, Morrow C, Lee N, Wood R. Decreasing household contribution to TB transmission with age: a retrospective geographic analysis of young people in a South African township. BMC Infect. Dis. 2014; 14(1): 221.

117. Zelner JL, Murray MB, Becerra MC, et al. Age-specific risks of tuberculosis infection from household and community exposures and opportunities for interventions in a high-burden setting. Am. J. Epidemiol. 2014; 180(8): 853-61.

118. Horna-Campos OJ, Consiglio E, Sanchez-Perez HJ, Navarro A, Cayla JA, Martin-Mateo M. Pulmonary tuberculosis infection among workers in the informal public transport sector in Lima, Peru. Occup. Environ. Med. 2011; 68(2): 163-5.

119. Escombe AR, Huaroto L, Ticona E, et al. Tuberculosis transmission risk and infection control in a hospital emergency department in Lima, Peru. Int. J. Tuberc. Lung Dis. 2010; 14(9): 1120-6.

120. Gandhi NR, Weissman D, Moodley P, et al. Nosocomial transmission of extensively drugresistant tuberculosis in a rural hospital in South Africa. J. Infect. Dis. 2013; 207(1): 9-17.

121. Dowdy DW, Golub JE, Chaisson RE, Saraceni V. Heterogeneity in tuberculosis transmission and the role of geographic hotspots in propagating epidemics. Proc. Natl. Acad. Sci. U. S. A. 2012; 109(24): 9557-62.

122. Jenkins HE, Gegia M, Furin J, et al. Geographical heterogeneity of multidrug-resistant tuberculosis in Georgia, January 2009 to June 2011. Euro Surveill. 2014; 19(11). 
123. Zelner JL, Murray MB, Becerra MC, et al. Identifying Hotspots of Multidrug-Resistant Tuberculosis Transmission Using Spatial and Molecular Genetic Data. J. Infect. Dis. 2015.

124. Blower SM, Gerberding JL. Understanding, predicting and controlling the emergence of drugresistant tuberculosis: a theoretical framework. J. Mol. Med. (Berl.) 1998; 76(9): 624-36.

125. Dye C, Espinal MA. Will tuberculosis become resistant to all antibiotics? Proc. Biol. Sci. 2001; 268(1462): 45-52.

126. Anderson RM, May RM. Infectious diseases of humans : dynamics and control. Oxford ; New York: Oxford University Press; 1991.

127. Cohen T, Dye C, Colijn C, Williams B, Murray M. Mathematical models of the epidemiology and control of drug-resistant TB. Expert Rev. Respir. Med. 2009; 3(1): 67-79.

128. Cohen T, Sommers B, Murray M. The effect of drug resistance on the fitness of Mycobacterium tuberculosis. Lancet Infect. Dis. 2003; 3(1): 13-21.

129. Comas I, Borrell S, Roetzer A, et al. Whole-genome sequencing of rifampicin-resistant Mycobacterium tuberculosis strains identifies compensatory mutations in RNA polymerase genes. Nat. Genet. 2012; 44(1): 106-10.

130. de Vos M, Muller B, Borrell S, et al. Putative compensatory mutations in the rpoC gene of rifampin-resistant Mycobacterium tuberculosis are associated with ongoing transmission. Antimicrob. Agents Chemother. 2013; 57(2): 827-32.

131. Ioerger TR, Feng Y, Chen X, et al. The non-clonality of drug resistance in Beijing-genotype isolates of Mycobacterium tuberculosis from the Western Cape of South Africa. BMC Genomics 2010; 11: 670 .

132. Casali N, Nikolayevskyy V, Balabanova Y, et al. Evolution and transmission of drug-resistant tuberculosis in a Russian population. Nat. Genet. 2014; 46(3): 279-86.

133. Grandjean L, Gilman RH, Martin L, et al. Transmission of Multidrug-Resistant and DrugSusceptible Tuberculosis within Households: A Prospective Cohort Study. PLoS Med. 2015; 12(6): e1001843; discussion e.

134. Shah S. Majority of XDR TB Cases are due to Transmission in a High HIV Prevalence Setting. 22nd Conference of Retroviral and Opportunistic Infections (23-26 Feb); 2015 23-26 Feb; Seattle; 2015.

135. Knight GM, Colijn C, Shrestha S, et al. The Distribution of Fitness Costs of Resistance-Conferring Mutations Is a Key Determinant for the Future Burden of Drug-Resistant Tuberculosis: A Model-Based Analysis. Clin. Infect. Dis. 2015; 61Suppl 3: S147-54.

136. Corbett EL, Charalambous S, Fielding K, et al. Stable incidence rates of tuberculosis (TB) among human immunodeficiency virus (HIV)-negative South African gold miners during a decade of epidemic HIV-associated TB. J. Infect. Dis. 2003; 188(8): 1156-63.

137. Corbett EL, Bandason T, Cheung YB, et al. Epidemiology of tuberculosis in a high HIV prevalence population provided with enhanced diagnosis of symptomatic disease. PLoS Med. 2007; 4(1): e22.

138. Huang CC, Tchetgen ET, Becerra MC, et al. The effect of HIV-related immunosuppression on the risk of tuberculosis transmission to household contacts. Clin. Infect. Dis. 2014; 58(6): 765-74.

139. Yates TA, Abubakar I, Tanser F. HIV Infection and the Transmission of Tuberculosis. J. Infect. Dis. 2014.

140. Corbett EL, Charalambous S, Moloi VM, et al. Human immunodeficiency virus and the prevalence of undiagnosed tuberculosis in African gold miners. Am. J. Respir. Crit. Care Med. 2004; 170(6): 673-9.

141. Group ISS, Lundgren JD, Babiker AG, et al. Initiation of Antiretroviral Therapy in Early Asymptomatic HIV Infection. N. Engl. J. Med. 2015; 373(9): 795-807.

142. Group TAS, Danel C, Moh R, et al. A Trial of Early Antiretrovirals and Isoniazid Preventive Therapy in Africa. N. Engl. J. Med. 2015; 373(9): 808-22.

143. WHO. Guideline on when to start antiretroviral therapy and pre-exposure prophylaxis for HIV. http://apps.who.int/iris/bitstream/10665/186275/1/9789241509565 eng.pdf. Geneva: World Health Organisation; 2015 [Accessed on 13/10/2015]. 
144. Lawn SD, Wood R, De Cock KM, Kranzer K, Lewis JJ, Churchyard GJ. Antiretrovirals and isoniazid preventive therapy in the prevention of HIV-associated tuberculosis in settings with limited health-care resources. Lancet Infect. Dis. 2010; 10(7): 489-98.

145. Lawn SD, Harries AD, Williams BG, et al. Antiretroviral therapy and the control of HIVassociated tuberculosis. Will ART do it? Int. J. Tuberc. Lung Dis. 2011; 15(5): 571-81.

146. Suthar AB, Lawn SD, del Amo J, et al. Antiretroviral therapy for prevention of tuberculosis in adults with HIV: a systematic review and meta-analysis. PLoS Med. 2012; 9(7): e1001270.

147. Middelkoop K, Wood R, Bekker LG. The impact of antiretroviral treatment programs on tuberculosis notification rates. Int. J. Tuberc. Lung Dis. 2011; 15(12): 1714; author reply -5.

148. Zachariah R, Bemelmans M, Akesson A, et al. Reduced tuberculosis case notification associated with scaling up antiretroviral treatment in rural Malawi. Int. J. Tuberc. Lung Dis. 2011; 15(7): 933-7. 149. Munthali L, Khan PY, Mwaungulu NJ, et al. The effect of HIV and antiretroviral therapy on characteristics of pulmonary tuberculosis in northern Malawi: a cross-sectional study. BMC Infect. Dis. 2014; 14: 107.

150. van Halsema CL, Fielding KL, Chihota VN, et al. Brief Report: The Effect of Antiretroviral Therapy and CD4 Count on Markers of Infectiousness in HIV-Associated Tuberculosis. J. Acquir. Immune Defic. Syndr. 2015; 70(1): 104-8.

151. Williams BG, Granich R, De Cock KM, Glaziou P, Sharma A, Dye C. Antiretroviral therapy for tuberculosis control in nine African countries. Proc. Natl. Acad. Sci. U. S. A. 2010; 107(45): 19485-9. 152. Pretorius C, Menzies NA, Chindelevitch L, et al. The potential effects of changing HIV treatment policy on tuberculosis outcomes in South Africa: results from three tuberculosis-HIV transmission models. AIDS 2014; 28 Suppl 1: S25-34.

153. Dodd PJ, Knight GM, Lawn SD, Corbett EL, White RG. Predicting the long-term impact of antiretroviral therapy scale-up on population incidence of tuberculosis. PLoS One 2013; 8(9): e75466. 


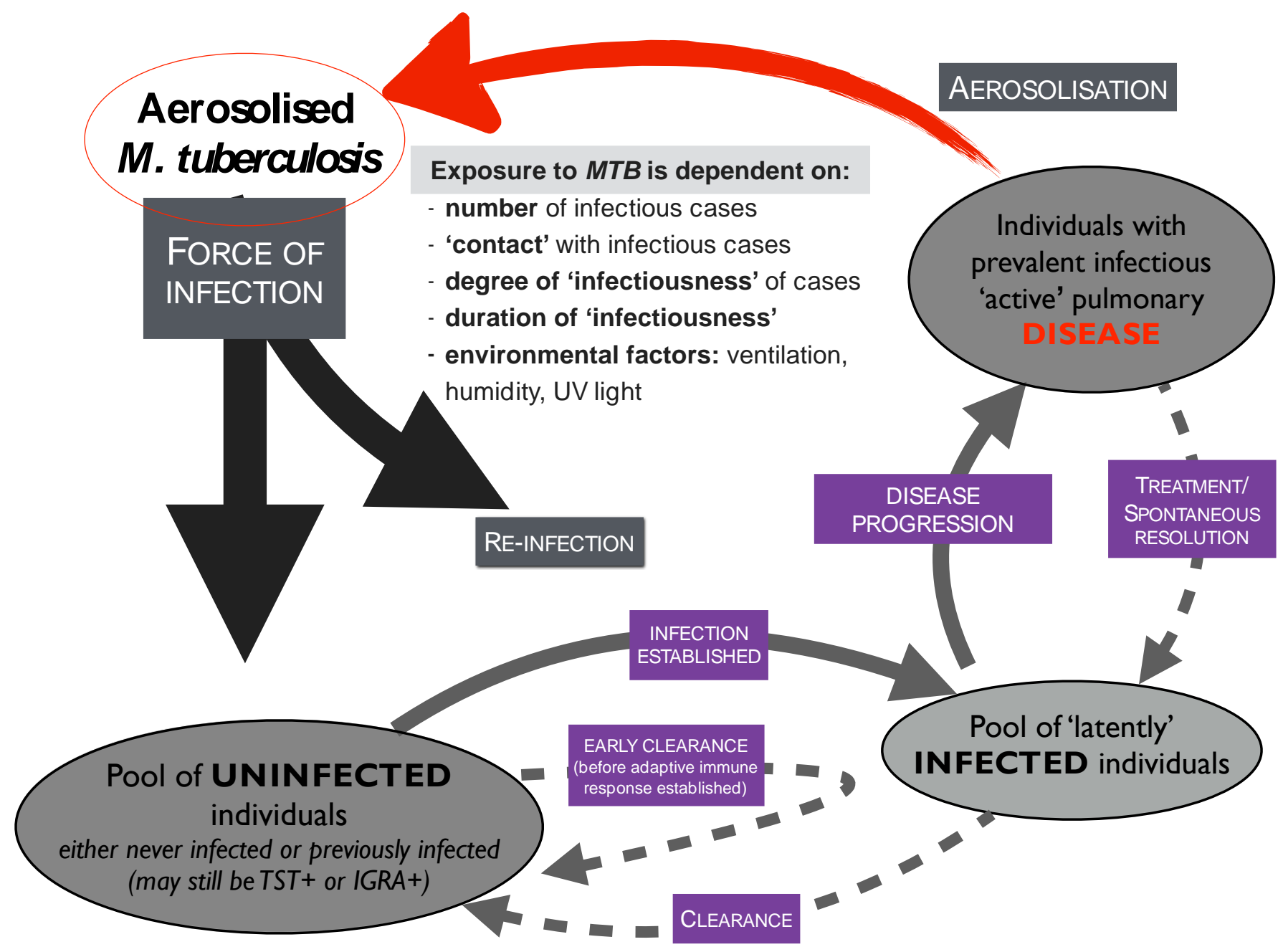

Figure 1. The MTB transmission cycle. 
Panel: Diagnostic tests of MTB infection

- There is no gold standard diagnostic test for MTB infection.

- The two widely used diagnostic tests are tuberculin skin tests (TSTs) and interferon-gamma release assays (IGRAs). Positive tests are interpreted as reflecting a prior adaptive immune response to mycobacterial infection.

- Currently it is not possible to detect infections cleared by the innate immune system prior to an adaptive response, ${ }^{41}$ nor to distinguish cleared infections that leave a lingering immunological footprint from persistent infection.

- Neither test is able to distinguish between latent infection and disease

Tuberculin skin test (TST)

- TSTs involve an intradermal injection of a standardised purified protein derivative (PPD) then measurement of any induration 48-96 hours later.

- Sensitivity and specificity are dependent on the number of millimetres of induration chosen as the cut point and the prevalence of non-specific reactions, which is determined by exposure to environmental mycobacteria and prior BCG vaccination. ${ }^{57}$ The BCG effect wanes in children vaccinated in infancy.

- Low sensitivity can be seen with advanced age and with immunosuppression as a result of malnutrition or HIV.

- New statistical techniques can suggest appropriate cut points for given distributions of reactions sizes. ${ }^{58}$

- Test reversions do occur and are commoner in young individuals, probably reflecting an initial false positive test. ${ }^{59}$

Interferon gamma release assay (IGRA)

- IGRAs involve a blood draw. T cells are then exposed to antigens that are found in MTB but not in BCG nor in most environmental mycobacteria.

- Interferon-gamma released by cells that recognise these antigens is then assayed in the supernatant following incubation (Quantiferon Gold) or by counting the number of interferon gamma producing cells in an ELISPOT assay (T Spot TB).

- IGRAs are a more specific test for MTB infection but there is less precedent for their use in transmission studies. The need for phlebotomy and the high cost of the test are also disadvantages.

- Test reversions are common and the clustering of results around the threshold for positivity means the choice of cut point can substantially affect sensitivity, specificity and prevalence estimates. ${ }^{15}$

New tools

- A new and, hopefully, more specific skin test based on similar antigens to those used in IGRAs is now being tested in Phase III trials. 60

- RNA expression signatures have been developed that may distinguish disease from latent infection, TB from other diseases and that might revert following successful treatment of active TB. ${ }^{61-63}$ These signatures require further validation. 
Table 1. Measures of MTB transmission in populations.

\begin{tabular}{|c|c|c|c|}
\hline Tool & What is measured & Advantages & Disadvantages \\
\hline $\begin{array}{l}\text { Prevalence of TB } \\
\text { infection }\end{array}$ & $\begin{array}{l}\text { Typically measured using TSTs in } \\
\text { school going children }\end{array}$ & $\begin{array}{l}\text { Cheap and well established } \\
\text { Infections must have occurred within an individual's lifetime, hence, in young children, } \\
\text { this is a measure of recent infection } \\
\text { Prevalence can be converted into an annualised incidence ('annual risk of tuberculosis } \\
\text { infection', ARTI) } \\
\text { Repeated surveys or continuous measurement of infection prevalence in the same age } \\
\text { group can quantify changes in MTB transmission over time }\end{array}$ & $\begin{array}{l}\text { Does not capture early clearance } \\
\text { Poor sensitivity and specificity, uncertainty regarding cut points plus conversions and reversions can } \\
\text { affect estimates in some populations } \\
\text { From a single study, it is not possible to separate age and cohort effects } \\
\text { 'Styblo's rule', which states that there is a fixed relationship between ARTI and the incidence of TB } \\
\text { disease, is no longer thought to be valid. } .^{64,65} \\
\text { WHO no longer recommend single tuberculin surveys. }\end{array}$ \\
\hline $\begin{array}{l}\text { Incidence of TB } \\
\text { infection }\end{array}$ & $\begin{array}{l}\text { Testing cohorts for } \mathrm{TB} \text { infection } \\
\text { longitudinally }\end{array}$ & $\begin{array}{l}\text { Older children can be included and inferences still made about recent transmission } \\
\text { An incidence cohort including older children and adults provides more general insights } \\
\text { into transmission in the community even if mixing patterns are strongly age assortative }\end{array}$ & $\begin{array}{l}\text { Does not capture early clearance } \\
\text { Requires larger sample or longer duration of follow up than measuring infection prevalence } \\
\text { Losses to follow up may reduce power and bias estimates } \\
\text { Excluding those who are positive at baseline may exclude those at highest risk - a particular problem } \\
\text { in older individuals in high burden settings }\end{array}$ \\
\hline TB notifications & $\begin{array}{l}\text { Notifications of TB disease to the } \\
\text { national treatment programme }\end{array}$ & $\begin{array}{l}\text { Data are routinely captured } \\
\text { It may be feasible to enhance capacity to diagnose and notify cases of TB for the purposes } \\
\text { of research though substantial biases and quality problems inherent to routinely collected } \\
\text { data are likely to persist }\end{array}$ & $\begin{array}{l}\text { Serious problems with data quality in most high burden settings } \\
\text { Only captures TB transmission that progresses to disease } \\
\text { Only captures individuals who access a diagnosis and whose diagnosis is notified } \\
\text { May capture individuals who do not have TB - poor specificity is a particular problem where TB } \\
\text { diagnosis is primarily based on chest X-ray such as in children }\end{array}$ \\
\hline $\begin{array}{l}\text { Prevalence of TB } \\
\text { disease }\end{array}$ & $\begin{array}{l}\text { Typically measured in large } \\
\text { surveys using sputum culture with } \\
\text { or without pre-screening for } \\
\text { symptoms and or with a chest x- } \\
\text { ray }\end{array}$ & $\begin{array}{l}\text { Well established } \\
\text { Undiagnosed individuals can be referred for treatment }\end{array}$ & $\begin{array}{l}\text { Substantial and expensive undertaking } \\
\text { Only captures TB transmission that progresses to (pulmonary) disease } \\
\text { It may not be clear whether changes in prevalence are a result of differences in transmission, in } \\
\text { progression from infection to disease, or in disease duration } \\
\text { Prevalence surveys are active case finding interventions and will transiently alter local TB } \\
\text { epidemiology } \\
\text { Sputum culture has low sensitivity in children }\end{array}$ \\
\hline $\begin{array}{l}\text { Incidence of TB } \\
\text { disease }\end{array}$ & $\begin{array}{l}\text { Measured in established cohorts or } \\
\text { using two prevalence surveys }\end{array}$ & Allows changes in incidence to be disaggregated from changes in disease duration & $\begin{array}{l}\text { Except in established cohorts in high burden settings, the measurement of incidence requires more } \\
\text { than one large prevalence survey - this is rarely feasible } \\
\text { Only captures TB transmission that progresses to (pulmonary) disease } \\
\text { It may not be clear whether differences in prevalence are a result of differences in transmission or in } \\
\text { progression from infection to disease }\end{array}$ \\
\hline $\begin{array}{l}\text { Molecular } \\
\text { epidemiology } \\
\text { (proportion } \\
\text { clustered) }\end{array}$ & $\begin{array}{l}\text { The proportion of isolates that } \\
\text { have the same strain type usually } \\
\text { using RFLP, MIRU-VNTR or WGS }\end{array}$ & $\begin{array}{l}\text { Allows inferences to be made about the proportion of TB resulting from reactivation vs. } \\
\text { recent infection } \\
\text { Strain typing can disprove or provide evidence to support putative transmission events }\end{array}$ & $\begin{array}{l}\text { Requires advanced laboratory capacity } \\
\text { Only captures transmissions that progress to disease and isolates that are sampled } \\
\text { Biased estimates can be obtained if the sampling fraction is low, if the study is not of sufficient } \\
\text { duration or if there is substantial in or out migration }\end{array}$ \\
\hline
\end{tabular}

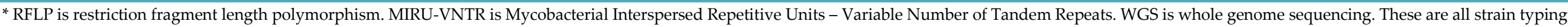
techniques. 